\section{Different types of amyloid concomitantly present in the same patients}

Francesca Martini, Gabriele Buda, Vincenzo De Tata, Sara Galimberti, Enrico Orciuolo, Matilde Masini, Mario Petrini

Department of Clinical and

Experimental Medicine, U.O. Hematology, University of Pisa, Italy

\section{Abstract}

Different types of amyloid concomitantly present in the same patient is believed to be improbable. We reported four cases of patients with plasma cell disorders who were found to have biopsy proven concomitant different types of amyloid fibrils deposition. We characterized amyloid fibrils using immunogold electron microscopy. There is lack of experience in the treatment of these frail and elderly patients, who are on the threshold between necessity of chemotherapy for AL amyloidosis and necessity to avoid harmful treatment related toxicity. All patients with a systemic amyloid syndrome require therapy to prevent deposition of amyloid in other organs and prevent progressive organ failure. Stem cell transplant (SCT) is preferred, but only $20 \%$ of patients are eligible Requirements for safe SCT include systolic blood pressure $>90 \mathrm{~mm} \mathrm{Hg}$, troponin $\mathrm{T}$ $<0.06 \mathrm{ng} / \mathrm{mL}$, age $<70$ years, and serum creatinine $\leq 1.7 \mathrm{mg} / \mathrm{dL}$ Nontransplant candidates can be offered melphalandexamethasone

cyclophosphamide-bortezomib-

dexamethasone.

\section{Introduction}

Amyloidosis covers a group of systemic disorders characterized by the deposition of misfolded protein within the extracellular space, with subsequent deposition in tissues and organ damage. The diagnostic approach is based on two fundamental steps: i) The identification of amyloid in bioptic samples (although testing for amyloid deposits by Congo red staining under polarized light microscopy is generally considered as the diagnostic gold standard, this method lacks in sensitivity and specificity; ii) The typing of amyloid fibrils in order to identify the chemical nature of the amyloidogenic protein. ${ }^{1-6}$ At our Centre the characterization of amyloid fibrils is achieved by immunogold electron microscopy. Immunogold labeling is a classic highresolution method that enables the selective localization of macromolecules in biological samples observed at the electron microscope by the use of specific antibodies coupled with colloidal gold particles. Clinically, the presence of amyloidosis in patients with plasma cell dyscrasia is usually assumed to be AL amyloidosis. We reported four cases of patients with plasma cell disorders who were found to have biopsy proven concomitant distinct type of amyloid fibrils deposition. Typing of amyloid deposits has significant implications in patient's management and prognosis.

\section{Materials and Methods}

We describe four patients, affected by monoclonal gammopathy, who underwent fat pad biopsy, because of clinically suspected amyloidosis.

Tissue samples obtained by periumbilical fat biopsies were fixed with $2.5 \%$ (vol./vol.) glutaraldehyde in 0.1 $\mathrm{mmol} / \mathrm{L}$ cacodylate buffer, $\mathrm{pH} 7.4$ for $1 \mathrm{~h}$ at $4^{\circ} \mathrm{C}$, and then post fixed in $1 \%$ (vol./vol.) cacodylate-buffered osmium tetroxide for 2 $h$ at room temperature. Samples were dehydrated in a graded series of ethanol, transferred to propylene oxide and embedded in Epon-Araldite. Ultrathin sections (60-80 nm thick) were cut with a diamond knife, placed on formvar/carboncoated copper grids (200 mesh), stained with uranyl acetate and lead citrate and observed under a Zeiss 902 transmission electron microscope. Immunogold labeling for immunoglobulin light chains $\kappa$ and $\lambda$, seroalbumin $\mathrm{A}$, and trans-thyretin was performed according to Bendayan [Bendayan M. Double immunocytochemical labeling applying the protein A-gold technique. J Histochem Cytochem 1982; 30: 81-85] by the use of guinea pig specific primary antibodies (Agilent Technologies Italia S.p.A., Cernusco sul Naviglio, Milano, Italy), together with protein A conjugated gold particles sized $15 \mathrm{~nm}$ (Agar Scientific, Stansted, UK). When deposits of TTR amyloid were found, we screened for genetic mutations to discriminate between hereditary (hATTR) and wild type forms of the disease (wtATTR).

\section{Case Report \#1}

A 73-year-old Caucasian male presented with longstanding chronic kidney disease
Correspondence: Francesca Martini, Department of Clinical and Experimental Medicine, U.O. Hematology, University of Pisa, Via Roma 67, 56100, Pisa, Italy.

Tel.: +39-3394470869

E-mail: m_88_f@libero.it

Key words: Amyloidosis, plasma cell dyscrasia, electron microscopy, multiple myeloma, rheumatologic disease, cardiovascular disease, renal disease.

Acknowledgements: Important contribution to the research by electron microscopy laboratory staff.

Contributions: FM and GB: was responsible for the study concept and design; FM and VT: analyzed and interpreted the data; FM: drafted the manuscript; and all authors: critically read and approved the final manuscript.

Conflict of interest: The authors declare no potential conflict of interest.

Funding: None.

Received for publication: 22 January 2019.

Accepted for publication: 7 June 2019.

This work is licensed under a Creative Commons Attribution-NonCommercial 4.0 International License (CC BY-NC 4.0).

(C) Copyright: the Author(s), 2019

Licensee PAGEPress, Italy

Hematology Reports 2019; 11:7996

doi:10.4081/hr.2019.7996

(CKD) G2 related to arterial hypertension stage II. Past medical history: prostate cancer and monoclonal gammopathy of undetermined significance (MGUS) IgG k, rosacea dermatitis, right hand arthritis.

Laboratory tests (Table 1) showed normal complete blood count (CBC), normal serum calcium value, creatinine $1.4 \mathrm{mg} / \mathrm{dL}$, B type natriuretic peptide (BNP) $161 \mathrm{pg} / \mathrm{mL}$, proteinuria $946 \mathrm{mg} / 24 \mathrm{~h}$, albuminuria 200 $\mathrm{mg} / \mathrm{dL}$. His serum protein electrophoresis (SPEP) and immunofixation (IFE) revealed a monoclonal IgG k M-protein at $2 \mathrm{~g} / \mathrm{dL}$. Twenty-four-hour urine protein electrophoresis (UPEP) demonstrated positive $\mathrm{k}$ light chain proteinuria, Bence Jones Proteinuria (BJP). Kappa serum free light chain (FLC) was $126 \mathrm{mg} / \mathrm{dL}$ (normal range $0,330-1,940 \mathrm{mg} / \mathrm{dL}$ ) and lambda serum FLC was $0.858 \mathrm{mg} / \mathrm{dL}$ (normal range $0.571-2.630 \mathrm{mg} / \mathrm{dL}$ ) with $\mathrm{kappa} / \mathrm{lambda}$ ratio at 146 (normal range 0.26-1.65).

Bone skeletal survey didn't detect osteolytic bone lesion.

Bone marrow biopsy revealed 
plasmocytosis with scatted CD138+ plasma cells at $30 \%$ (Congo red staining not evaluated). We assessed amyloidosis as a concomitant CKD's cause: Congo red staining test on fat pad biopsy was positive. Immunogold on fat pad sample revealed transthyretin (ATTR) and serum amyloid A (SAA) amyloidosis. We screened for genetic mutations: the patient was affected by wild type form of the disease (wtATTR) (Figure $1)$.

The patient didn't have systemic AL amyloidosis. The value of FLC ratio above 100 was confirmed in serial testing. This is an IMWG criteria for treatment (FLC ratio $>100$ with concomitant FLC $\mathrm{k}>100 \mathrm{mg} / \mathrm{L}$ ), but in this case, treatment was delayed and FLC ratio monitored.

In clinical practice it is a common error to assume AL primary amyloidosis in patients presenting with monoclonal gammopathy. Rheumatologic assessment in order to identify underlying cause of SAA amyloid will be needed, considering patient history of rosacea dermatitis and hand arthritis. As a result of the correct diagnosis being made, the prognosis was much more favorable. It is known the high prevalence of coexistent MGUS with ATTR amyloidosis in the elderly population. The incidence of MGUS in wild type TTR cardiac amyloidosis is $23 \% ;^{7,8}$ moreover, it could be not uncommon to discover a plasma cell disorder in patients with SAA amyloid, given the increasing incidence of both conditions with chronic inflammatory disease.

\section{Case Report \#2}

A 70-year-old Caucasian male presented with CKD G3 related to arterial hypertension stage II. Past medical history: cardiac diastolic dysfunction New York Heart Association (NYHA) class II, mitral insufficiency and pulmonary hypertension; hypercholesterolemia and coronary artery atherosclerosis without hemodynamically significant stenosis.

The patient complained about dysgeusia, hyporexia and severe weight loss $(10 \mathrm{~kg}$ in 6 months); a chronic antral gastritis had been diagnosed by gastroscopy.

Laboratory tests (Table 1) showed normocitic anaemia (11.9 g/dL), normal serum calcium value, creatinine $1.36 \mathrm{mg} / \mathrm{dL}$,
N-terminal prohormone of brain natriuretic peptide (NT pro BNP) $7400 \mathrm{pg} / \mathrm{mL}$, proteinuria $975 \mathrm{mg} / 24 \mathrm{~h}$, albuminuria 70 $\mathrm{mg} / \mathrm{dL}$. His serum protein electrophoresis (SPEP) and immunofixation (IFE) revealed a monoclonal IgG lambda M-protein at 0.6 $\mathrm{g} / \mathrm{dL}$. Twenty-four-hour urine protein electrophoresis (UPEP) demonstrated positive lambda light chain proteinuria, Bence Jones Proteinuria (BJP). Kappa serum FLC was $1.740 \mathrm{mg} / \mathrm{dL}$ (normal range 0.330$1.940 \mathrm{mg} / \mathrm{dL}$ ) and lambda serum FLC was 8.240 (normal range $0.571-2.630 \mathrm{mg} / \mathrm{dL}$ ) with kappa/lambda ratio at 0.211 (normal range 0.26-1.65).

Echocardiogram estimated the 2D global left ventricular ejection fraction at $55 \%$; severe biventricular hypertrophy; diastolic

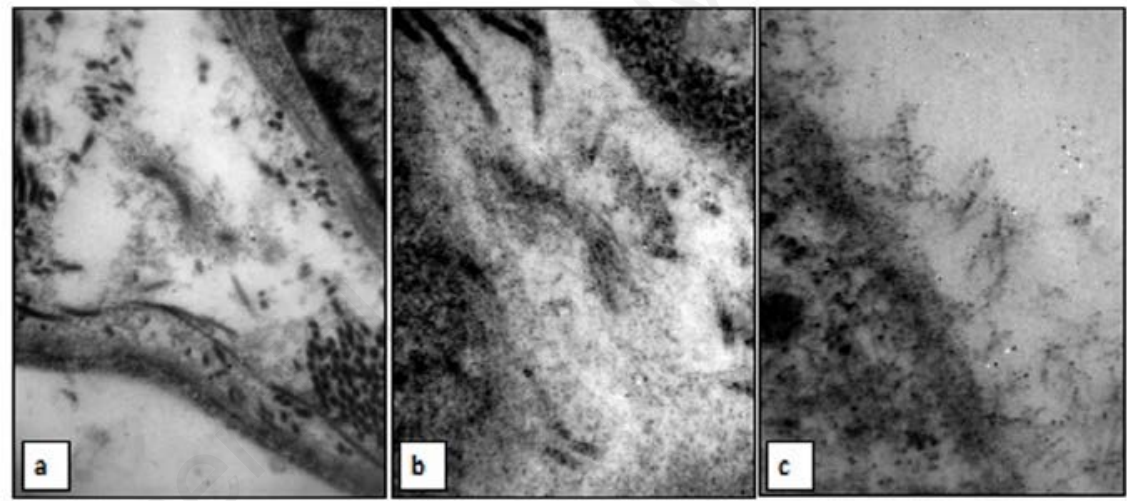

Figure 1. Case 1 (a) electron microscopy assay of fat pad biopsy: amyloid fibrils; (b) immunogold (5 $\mathrm{nm}$ ) transthyretin (ATTR) and (c) serum amyloid A (SAA) amyloidogenic deposition.

Table 1. Patients' characteristics.

\begin{tabular}{|c|c|c|c|c|}
\hline & Case 1 & Case 2 & Case 3 & Case 4 \\
\hline Age (years) & 73 & 70 & 76 & 80 \\
\hline Gender & Male & Male & Male & Male \\
\hline Clinical presentation & $\begin{array}{l}\text { CKD G2 arterial hypertension } \\
\text { stage II }\end{array}$ & $\begin{array}{l}\text { CKD G3 arterial hypertension } \\
\text { stage II; cardiac diastolic } \\
\text { dysfunction NYHA II; dysgeusia, } \\
\text { hyporexia, weight loss }\end{array}$ & CKD G3 arterial hypertension stage II & $\begin{array}{l}\text { CKD G2 arterial hypertension } \\
\text { stage III; peripheral neuropathy; } \\
\text { congestive heart failure NYHA II; } \\
\text { smoldering myeloma }\end{array}$ \\
\hline M protein (g/dL) & $\lg G$ k (2) & $\operatorname{IgGL}(0.6)$ & $\operatorname{IgM} k(1.25)$ & $\operatorname{IgG~k}(1.5)$ \\
\hline Kappa/lambda ratio & 146 & 0.211 & 3.71 & 1.83 \\
\hline $\begin{array}{l}\text { Value of the involved } \\
\text { light chain, } \mathrm{mg} / \mathrm{dL}\end{array}$ & 126 & 8.240 & 9.020 & $4.5^{*}$ \\
\hline BM biopsy & $30 \%$ plasma cells & $10 \%$ plasma cells & Not done & $10-12 \%$ plasma cells \\
\hline Organ biopsy & Not done & Endomyocardial biopsy & Not done & Not done \\
\hline Fat pad biopsy & ATTR and SAA amyloid & $\begin{array}{l}\text { ATTR and AL (lambda light chain) } \\
\text { amyloid }\end{array}$ & AL (k light chain) and SAA amyloid & $\begin{array}{l}\text { ATTR and AL (lambda light chain) } \\
\text { amyloid }\end{array}$ \\
\hline Diagnosis & $\begin{array}{l}\text { ATTR and SAA amyloidosis } \\
\text { and multiple myeloma }\end{array}$ & ATTR and AL amyloidosis & AL and SAA amyloidosis & $\begin{array}{l}\text { ATTR and AL (lambda light chain) } \\
\text { amyloidosis }\end{array}$ \\
\hline Management & Follow-up & Mel-Dex + doxycycline & Follow-up & Follow-up \\
\hline
\end{tabular}


dysfunction grade 2 (restrictive filling pattern). Cardiac MRI (magnetic resonance imaging) revealed features suggesting cardiac amyloidosis (severe biventricular hypertrophy). Tc $99 \mathrm{~m}$ pyrophosphate scan was diagnostic for cardiac ATTR amyloidosis. $^{9}$

Bone marrow biopsy revealed plasmocytosis with scatted CD138+ plasma cells at $10 \%$. Congo red staining on bone marrow, endomyocardial and fat pad biopsy were positive. Immunohistochemistry (ICH) staining on fat pad and on endomyocardial biopsy samples revealed ATTR and AL amyloidosis (lambda light chain). Immunogold on fat pad and on endomyocardial biopsy samples revealed only $\mathrm{AL}$ amyloidosis. We screened for genetic mutations: the patient was affected by wild type form of the disease (wtATTR) (Figure 2).

Unfortunately, gastric tissue had not been biopsied, so we couldn't confirm suspected soft tissue involvement, resulting in dysgeusia.

It was a challenging diagnostic case: after an in-depth discussion with pathologists patient was diagnosed with wtATTR and AL amyloidosis, supported by Tc $99 \mathrm{~m}$ pyrophosphate scan and ICH (even if we know that immunohistochemistry is not a reliable typing method unless done by very expert amyloid pathologist with very specific antibody panels); he was classified as intermedium risk according to "Guidelines 2011-2012 for Amyloidosis University of Pavia" and as stage III according to Mayo Clinic staging system. The presence of concomitant ATTR cardiac amyloidosis could represent an interfering factor for the use Mayo clinic staging system.

He was treated with melphalan 0,14 $\mathrm{mg} / \mathrm{kg}$ day 1-4 and dexamethasone $40 \mathrm{mg}$ day 1-4 (Mel-Dex) for six 28 days-cycles. Doxycycline therapy was added $(100 \mathrm{mg}$ bis a die-bid-) because doxycycline combined with tauroursodeoxycholic acid improved cardiac outcomes in transthyretin amyloidosis and in a cohort of patients with systemic AL amyloidosis, doxycycline, given as antimicrobial prophylaxis post autologous stem cell transplantation, significantly improved survival compared to controls who were given penicillin. The main impact of doxycycline is reduction in early cardiac mortality. ${ }^{10}$ Treatment of AL amyloidosis is not similar to MM in terms of the duration of treatment and these patients should be treated of 2 cycles past best response. We avoided bortezomib because it could be associated with worsening fluid retention and congestive symptoms. Disease assessment after 2 cycles was unsatisfactory, so the patient received 4 further cycles of chemotherapy. Disease assessment after 6 cycles of chemotherapy revealed a hematological partial response (dFLC reduction $>50 \%$ ), as well as steady heart and renal functions, however patient still complained dysgeusia and dyspnea. Considering potential chemotherapy toxicity, harmful to senile amyloidosis component, we stopped chemotherapy and the patient



Figure 2. Case 2 (a) electron microscopy assay of fat pad biopsy: amyloid fibrils; (b) immunogold (20 nm) lambda light chains (AL); (c) endomyocardial biopsy immunogold $(20 \mathrm{~nm})$ lambda light chains amyloidogenic deposition.
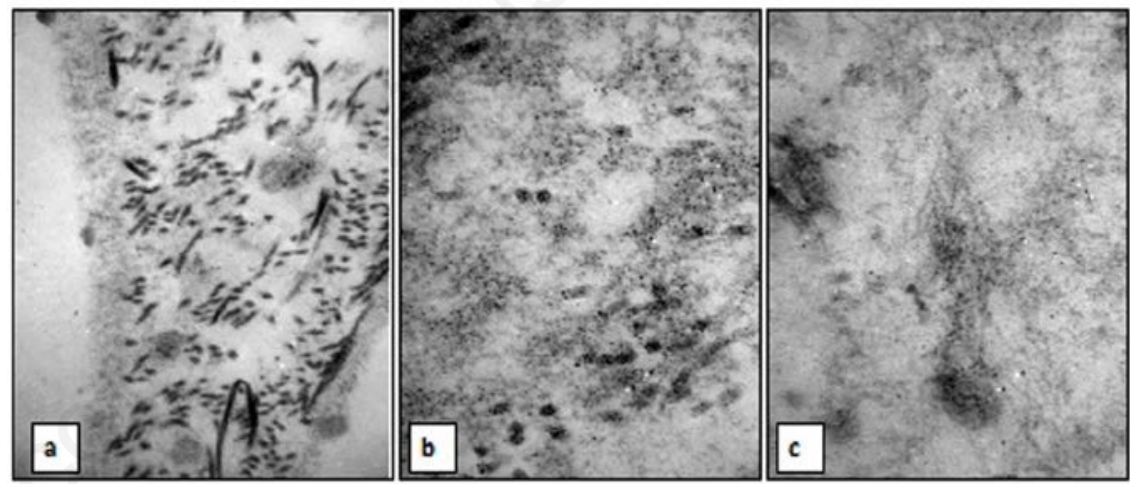

Figure 3. Case 3 (a) electron microscopy assay of fat pad biopsy: amyloid fibrils (b) immunogold $(5 \mathrm{~nm}) \mathrm{k}$ light chain (AL) and (c) serum amyloid A (SAA) amyloidogenic deposition.
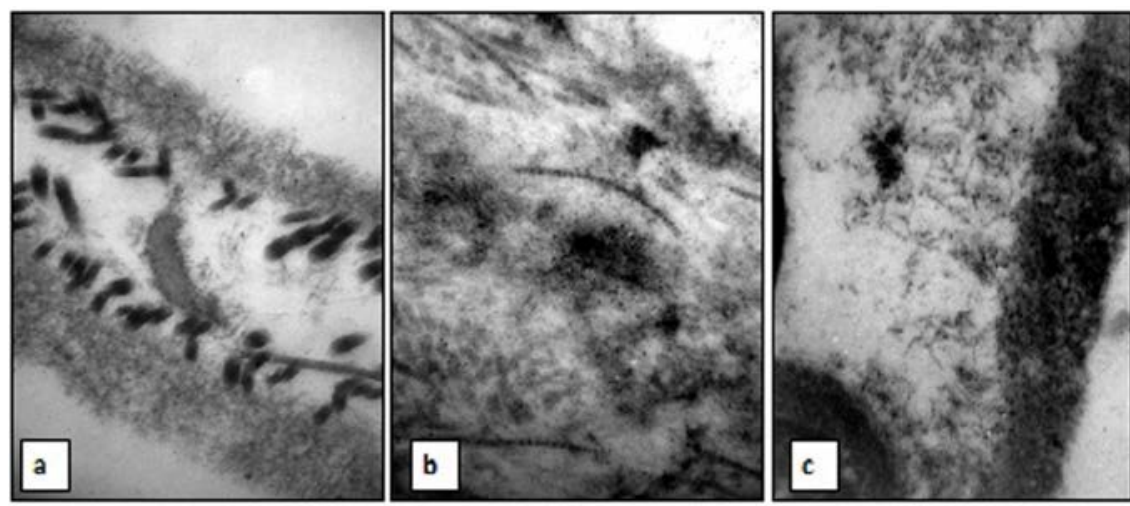

Figure 4. Case 4 (a) electron microscopy assay of fat pad biopsy: amyloid fibrils (b) immunogold (5 nm) lambda light chains (AL) and (c) transthyretin (ATTR) amyloidogenic deposition. 
was closely monitored with both clinical and laboratory follow up. After three months from the end of chemotherapy treatment, the patient developed an acute heart failure. He was hospitalized and treated with diuretics, obtaining clinical status improvement. Considering the worsening cardiac function, the increasing in lambda FLC value, and the previous good response obtained with MelDex, the patient was treated with Mel-Dex again (3 cycles) obtaining a mild clinical picture improvement.

In case of disease progression non cardiotoxic immunomodulatory drug, IMIDs, could be administered.

\section{Case Report \#3}

A 76-year-old Caucasian male presented with CKD G3 related to arterial hypertension stage II. Past medical history: hepatitis C virus (HCV) positivity, MGUS $\operatorname{IgM~k}$, hepatic steatosis, hypertensive heart disease, type 2 diabetes mellitus, prostatic hypertrophy. Physical examination revealed lower limb oedema (related to heart dysfunction).

Laboratory tests (Table 1) showed normocitic anaemia $(11.0 \mathrm{~g} / \mathrm{dL})$, normal serum calcium value, creatinine $3.30 \mathrm{mg} / \mathrm{dL}$, B type natriuretic peptide (BNP) $330 \mathrm{pg} / \mathrm{mL}$, proteinuria $6138 \mathrm{mg} / 24 \mathrm{~h}$, albuminuria 200 $\mathrm{mg} / \mathrm{dL}$. His serum protein electrophoresis (SPEP) and immunofixation (IFE) revealed a monoclonal IgM k M-protein at $1.25 \mathrm{~g} / \mathrm{dL}$. Twenty-four-hour urine protein electrophoresis (UPEP) was negative. Kappa serum free light chain (FLC) was 9.020 $\mathrm{mg} / \mathrm{dL}$ (normal range $0.330-1.940 \mathrm{mg} / \mathrm{dL}$ ) and lambda serum FLC was 2.430 (normal range $\quad 0.571-2.630 \quad \mathrm{mg} / \mathrm{dL}$ ) with kappa/lambda ratio at 3.710 (normal range $0.26-1.65)$.

We assessed amyloidosis as a concomitant CKD's and heart disease's cause: Congo red staining test on fat pad biopsy was positive. Immunogold on fat pad sample revealed AL (k light chain) and SAA amyloidosis. The patient refused bone marrow exam (Figure 3).

We decided not to treat the patient, considering the low FLC $\mathrm{k}$ value and the concomitant SAA amyloid, that couldn't benefit from chemotherapy. The patient is being followed up every three months to detect promptly any free light chain gain or organ impairment worsening.

Underlying cause of SAA amyloidosis probably was long standing HCV chronic infection. No rheumatic diseases could be reported after rheumatologic investigations.

\section{Case Report \#4}

An 80-year-old Caucasian male presented with CKD G2, arterial hypertension stage III, peripheral neuropathy, congestive heart failure (NYHA-II). Past medical history: heart attack, dyslipidemia, lung cancer, diabetes mellitus caused by recurrent acute pancreatitis of undetermined nature and $\mathrm{IgG}$ $\mathrm{k}$ smouldering myeloma.

Laboratory tests (Table 1) showed mild normocytic anemia (11.5 g/dL), normal serum calcium value, creatinine $2.21 \mathrm{mg} / \mathrm{dL}$, NT-proBNP $2.337 \mathrm{ng} / \mathrm{L}$, high sensitivity cardiac troponin (HS-cTn) was $76 \mathrm{ng} / \mathrm{L}$, albuminuria $93 \mathrm{mg} / \mathrm{dL}$. His serum protein electrophoresis (SPEP) and immunofixation (IFE) revealed a monoclonal IgG k Mprotein at $1.5 \mathrm{~g} / \mathrm{dL}$. Twenty-four-hour urine protein electrophoresis (UPEP) was negative (negative BJP). Kappa serum free light chain (FLC) was $4.5 \mathrm{mg} / \mathrm{dL}$ (normal range 0.330$1.940 \mathrm{mg} / \mathrm{dL}$ ) and lambda serum FLC was $2.4 \mathrm{mg} / \mathrm{dL}$ (normal range 0.571-2.630 $\mathrm{mg} / \mathrm{dL}$ ) with kappa/lambda ratio at 1.830 (normal range 0.26-1.65).

Echocardiogram estimated the 2D global left ventricular ejection fraction at $58 \%$. There was mild left ventricular hypertrophy with increased septal thickness and myocardial mass index, diastolic dysfunction grade 1, suggesting cardiac amyloidosis. Cardiac magnetic resonance imaging with gadolinium contrast was contraindicated because of moderate kidney disease. ${ }^{11}$

Congo red staining on fat pad biopsy was positive. Immunogold on fat pad sample revealed ATTR and lambda AL amyloidosis. We screened for genetic mutations: the patient was affected by wild type form of the disease (wtATTR) (Figure 4).

Clinical picture (heart, kidney, peripheral nervous systems damage) was in accord with systemic AL amyloidosis and could benefit from chemotherapy. Concomitant presence of wtATTR, that doesn't benefit from chemotherapy and the recent history of lung cancer advised against chemotherapy, if not really necessary. In order to improve neurological symptoms steroid therapy could be administered, avoiding harmful chemotherapic toxicity, but also this therapy could be potentially toxic because of concomitant poorly controlled diabetes mellitus. Therefore, the patient was closely followed up.

Lambda AL is three times more common than kappa AL. It is expected to find the same light chain clonal restriction both in $\mathrm{M}$ component and amyloid fibrils. However, this patient presented with an $\operatorname{IgG} \mathrm{k}$ M- protein, but immunogold on fat pad sample revealed lambda light chain amyloid fibrils. We could speculate about two distinct concomitant plasmacellular clones with different light chain restriction. There are very few data in medical literature.

\section{Discussion and Conclusions}

Accurate amyloid subtyping is extremely important in the management and prognosis of patients. Unfortunately, we couldn't use the gold standard laser capture mass spectroscopy, so the characterization of amyloid fibrils is achieved by immunogold electron microscopy. Concomitant detection of distinct type of amyloid fibrils is believed to be a rare event. Although the mechanism remains unclear, researchers speculate on a synergistic fibrilization of two type of amyloidogenic proteins. ${ }^{12}$ The method used does not allow evaluation as to whether there are two separate amyloid or if there are mixed forms. Combination with western blot and/or mass-spectrometry based proteomic characterization would make it possible.

A chemotherapic approach is necessary when AL amyloidosis is diagnosed, because of its inexorable progressive course due to uncontrolled organ damage. We described 4 cases characterized by different types of amyloid fibrils deposition concomitantly present in the same patient. We should be able to treat AL amyloidosis patients, avoiding harmful chemotherapic toxicity, because other concomitant amyloidosis types, like wtATTR, don't benefit from chemotherapy. ${ }^{13,14}$ Therefore, patients are closely monitored with both clinical and laboratory follow up, as long as we can. If chemotherapy is necessary we choose the less toxic drugs, along with supportive care according to organ damage if present. More clinical experience is needed to further optimize treatment-related toxicity and improve the outcome of these patients.

\section{References}

1. Gertz MA Immunoglobulin light chain amyloidosis: 2018 Update on diagnosis, prognosis, and treatment. Am J Hematol. 2018 ;93:1169-80

2. Nuvolone M, Merlini G. Systemic amyloidosis: novel therapies and role of biomarkers. Nephrol Dial Transplant 2017;32:770-80.

3. Merlini G, Seldin DC, Gertz MA. Amyloidosis: Pathogenesis and New Therapeutic Options. J Clin Oncol 2011;29:1924-33. 
4. Dispenzieri A, Buadi F, Kumar SK, et al. Treatment of Immunoglobulin Light Chain Amyloidosis: Mayo Stratification of Myeloma and Risk-Adapted Therapy (mSMART) Consensus Statement. Mayo Clin Proc 2015;90:1054-81.

5. Clement CG, Truong LD. An evaluation of Congo red fluorescence for the diagnosis of amyloidosis. Hum Pathol 2014;45:1766-72.

6. Castellani C, Fedrigo M, Frigo AC, et al. Application of confocal laser scanning microscopy for the diagnosis of amyloidosis. Virchows Arch 2017;470:455-63.

7. Phull P, Sanchorawala V, Connors LH. Monoclonal gammopathy of undetermined significance in systemic transthyretin amyloidosis (ATTR).
Amyloid 2018;25:62-7.

8. Geller HI, Singh A, Mirto TM, et al. Prevalence of Monoclonal Gammopathy in Wild-Type Transthyretin Amyloidosis. Mayo Clin Proc 2017;92:1800-5.

9. Gillmore JD. Maureer MS, Falk RH, et al. Nonbiopsy diagnosis of cardiac transthyretin amyloidosis. Circulation 2016;133;2404-12.

10. Kumar SK, Dispenzieri A, Lacy MQ, et al Doxycycline used as post transplant antibacterial prophylaxis improves survival in patients with light chain amyloidosis undergoing autologous stem cell transplantation. Blood 2012;120:3138.

11. Kuo PH, Kanal E, Abu-Alfa AK, Cowper SE. Gadolinium-based MR contrast agents and nephrogenic systemic fibrosis. Radiology 2007;242:647-9.

12. Giasson BI, Forman MS, Higuchi M, et al. Initiation and Synergistic Fibrillization of Tau and AlphaSynuclein. Science 2003;300:636-40.

13. Roof L, Coker WJ, Lazarchick J, Kang Y. Senile transthyretin cardiac amyloidosis in patients with plasma cell dyscrasias: importance of cardiac biopsy for making the correct diagnosis. Aperito J Cell Mol Biol 2014;1:102.

14. Ng B, Connors LH, Davidoff R, et al. Senile systemic amyloidosis presenting with heart failure. A Comparison With Light Chain-Associated Amyloidosis. Arch Intern Med 2005;165:1425-9. 